\title{
Duration of Action of Insulin Products in Diabetic Mellitus Patients- Possible Implications on Bio-similarity Assessment
}

\section{Srinivas NR*}

Zydus Research Center, Sarkhej-Bavla NH 8A, Moraiya, Ahmedabad, 382210, Gujarat, India

As a clinical pharmacologist and drug developer in the intriguing and evolving field of biosimilars, I often introspect on the newly published scientific data as to what it means in the current scheme of things on the perceived expectations on the bio-similarity assessment. The key question that I always ponder is to what degree would that such newly published data may influence or alter the current thinking of regulatory decision makers. In this editorial, the case study of two insulin glargine products is discussed to set the context. The editorial aims to provide views and general introspection on the published data of the two insulin products which demonstrated the totality of evidence to confirm the similarity of the two insulin products in the expected clinical outcome.

Recently, Linnebjerg et al. carried out an interesting study of two insulin glargine products, comprising of reference (Lantus insulin; IGlar) vs. treatment (LY2963016; LY IGlar) in type 1 diabetes mellitus patients [1]. LY IGlar has been previously confirmed to be biosimilar to IGlar in terms of its pharmacokinetic and pharmacodynamic profiles in healthy volunteers [2]. In particular, such data appears to raise the bar in our understanding of what needs to be a scientifically relevant package for showing bio-similarity of insulin products. The present study was not designed with the intent to prove equivalence of the two products from a bio-similarity perspective on the duration of response; however, the objective of the study was to provide sufficient time duration for the two insulin glargine products to allow the blood glucose levels to reach the cut-off threshold limit of $>8.3 \mathrm{mmol} / \mathrm{L}(150 \mathrm{mg} / \mathrm{dL})$ in the type 1 diabetes mellitus patients [1].

The current bio-similarity assessment on insulin products hinges on the use of healthy volunteers for pharmacokinetic and pharmacodynamic equivalence of the test vs. reference products. Whereas the pharmacokinetic assessment employs comparison of standard parameters for absorption and exposure, the pharmacodynamic equivalence is typically achieved by Euglycaemic clamp study for a short duration (i.e., 24 h) [3]. Therefore, one key question to ask would be: Does the two products that show bio-similarity in pharmacokinetics and pharmacodynamics in healthy volunteers have therapeutic equivalence in the duration of response in the patients? To that end, the planned study in type 1 patients addresses the important component of duration of action by extending the Euglycaemic clamp duration to $42 \mathrm{~h}$ instead of the regular shorter $24 \mathrm{~h}$ clamp duration studies that are typically followed to assess the duration of action of certain insulin products $[1,3]$.

It is fair to ask the question whether in the actual therapeutic management of type 1 diabetics is it important to know the total duration of action of the insulin product? While this parameter may not have any attached clinical relevance for treating diabetic patients with insulin therapy at regular intervals as deemed fit, it is a good yard stick to account for in the similarity comparison of the two products to understand the purported duration of action of the two products. The duration of $42 \mathrm{~h}$ clamp study seems somewhat insurmountable task from an execution perspective given the various risks involved in such a laboratory procedure; however, the authors were able to achieve a successful completion of the study in $42 \mathrm{~h}$ for both products in majority of the patients [1]. Despite the efforts, the end of study cut-off point of $>8.3 \mathrm{mmol} / \mathrm{L}$ ( $150 \mathrm{mg} / \mathrm{dL}$ ) was not achieved in 14 out of the 40 clamps investigated in this clinical trial (seven apiece for the treatment and reference products) [1].

Another question to introspect here: is: How does this duration of action study fit in the overall scheme of insulin product positioning for treating diabetic patients? Because of the use of a rather low dose of insulin $(0.3 \mathrm{U} / \mathrm{kg})$, in order to have manageable time duration for the Euglycaemic clamp study, one would be deviating from the clinical or therapeutic dose from an efficacy perspective. Also, because the diabetic patients are likely to enter therapy at different starting insulin doses which continue to be titrated, the duration of action study that looks at a small fixed dose of insulin may not truly represent a significant value add from a clinical perspective.

One risk associated with the duration of action study of the insulin products is that what if differential outcome for the two biosimilar products (established bioequivalence using measures of pharmacokinetics and pharmacodynamics) arise which may not be attributable to product differences but due to host of other factors such as the study design, patient population, pre-medications, influence of endogenous insulin etc. Therefore, there is a small chance for a deemed biosimilar insulin product to be inferior to the comparator (reference) product in having a lesser duration of action or vice versa, if a statistically well-powered study is conducted.

The credence to difficulty in the assessment of duration of action is provided by the rather fluctuating data of blood glucose levels measured by the Euglycaemic clamp study [1]. Within $42 \mathrm{~h}$ time period predetermined for the parameter assessment, the mean blood glucose levels fluctuated between the reference vs. test treatment arms, with one product numerically dominating for the first $24 \mathrm{~h}$ and the other product numerically showing higher values for the remainder of the period (i.e., 24-42 h).

While Linnebjerg et al. are not arguing that an inclusion of duration of action study should be an exercise that needs to be part of the biosimilarity evidence, the compelling data provided in the report eluded to the fact that such data may be extremely beneficial in the positioning of the biosimilar insulin product vis-à-vis the innovator insulin product on the branding of the biosimilar product [1]. However,

*Corresponding author: Srinivas NR, PhD, FCP, Zydus Research Centre, Sarkhej-Bavla NH 8A, Moraiya, Ahmedabad 382 210, Gujarat, India, Tel: 9180 6662 8801; E-mail: nuggehally.srinivas@zyduscadila.com

Received January 28, 2017; Accepted February 01, 2017; Published February 20, 2017

Citation: Srinivas NR (2017) Duration of Action of Insulin Products in Diabetic Mellitus Patients-Possible Implications on Bio-similarity Assessment. J Bioequiv Availab 9: e75. doi:10.4172/jbb.10000e75

Copyright: @ 2017 Srinivas NR. This is an open-access article distributed under the terms of the Creative Commons Attribution License, which permits unrestricted use, distribution, and reproduction in any medium, provided the original author and source are credited. 
Citation: Srinivas NR (2017) Duration of Action of Insulin Products in Diabetic Mellitus Patients-Possible Implications on Bio-similarity Assessment. J Bioequiv Availab 9: e75. doi:10.4172/jbb.10000e75

Page 2 of 2

as Linnebjerg et al. have eloquently discussed it would still fall short of the requirement for the interchangeability of the biosimilar product with that of the innovator product [1].

In summary, Linnebjerg et al. needed to be appreciated for undertaking a $42 \mathrm{~h}$ Euglycaemic clamp study with the purpose of measuring the duration of response of insulin [1]. However, the application of such a study with all the caveats (lower doses, fluctuating blood glucose levels etc.), including data interpretation for such patients who do not hit the threshold cut-off point at the end of the $42 \mathrm{~h}$ time period for an approved biosimilar product need to be made with caution given the fact that lower doses of insulin used in such protocols are mainly driven by the objective to measure the total duration of action as opposed to the purported efficacy of the product. Hence, incorporation of a definitive measure of total duration of action for insulin products in bio-similarity assessment does not appear to be justified from a regulatory perspective.

\section{Conflict of Interest}

The author is an employee of Cadila Health Care Ltd. (Ahmedabad, India) The manuscript (ZRC Publication No. 508) was prepared with the intent of scientific exchange on an important topic in clinical pharmacology and drug development. The author has no conflict or competing interests to declare.

\section{References}

1. Linnebjerg H, Lam EC, Zhang X, Mary E, Seger MS, et al. (2017) Duration of action of two insulin glargine products, LY2963016 insulin glargine and Lantus insulin glargine, in subjects with type 1 diabetes mellitus. Diabetes Obes Metab 19: 33-39.

2. Linnebjerg H, Lam EC, Seger ME, Coutant D, Chua L, et al. (2015) Comparison of the pharmacokinetics and pharmacodynamics of LY2963016 insulin glargine and EU- and US-approved versions of Lantus insulin glargine in healthy subjects: three randomized euglycemic clamp studies. Diabetes Care 38 : 2226-2233.

3. European Medicines Agency (2015) Guideline on non-clinical and clinical development of similar biological medicinal products containing recombinant human insulin and insulin analogues. London, UK. 